\title{
Velocity Planning System Based on Intelligent Vehicle Infrastructure Cooperative Technology
}

\author{
${ }^{1}$ Changqing Dong, ${ }^{2}$ Chen Chen, ${ }^{3}$ Xiaowei Chen, ${ }^{4}$ Haibo Dong, ${ }^{5}$ Kaixin Yang, ${ }^{6}$ Song Li \\ ${ }^{1}$ Automotive Data Center, China Automotive Technology\&Research Center Co. Ltd, Tianjin China \\ ${ }^{2}$ Automotive Data Center, China Automotive Technology\&Research Center Co. Ltd, Tianjin China \\ ${ }^{3}$ Automotive Data Center, China Automotive Technology\&Research Center Co. Ltd, Tianjin China \\ ${ }^{4}$ Automotive Data Center, China Automotive Technology\&Research Center Co. Ltd, Tianjin China \\ ${ }^{5}$ Automotive Data Center, China Automotive Technology\&Research Center Co. Ltd, Tianjin China \\ ${ }^{6}$ Automotive Data Center, China Automotive Technology\&Research Center Co. Ltd, Tianjin China \\ ${ }^{1}$ dongchangqing@catarc.ac.cn, ${ }^{2}$ chenchen@catarc. ac.cn, ${ }^{3}$ chenxiaowei1030@ \\ 126.com, ${ }^{4} 13920587597 @ 163 . c o m,{ }^{5}$ yangkaixin@catarc.ac.cn, ${ }^{6}$ kingofmount@163.com
}

Keywords: velocity planning algorithm, Intelligent vehicle infrastructure cooperative, ZigBee, road side unit, on board unit

\begin{abstract}
Travel time, fuel consumption and carbon dioxide emissions are directly related to the vehicle velocity. In this paper, we aim to give a velocity planning system based on intelligent vehicle infrastructure cooperative technology. The system contains two units: road side unit (RSU) and on board unit (OBU). RSU and OBU exchange information with ZigBee protocol. RSU gives dynamic speed advice to OBU by velocity planning algorithm so that the probability of having a green phase is maximized when approaching signalized intersections and the vehicle never exceeds the speed limit and it will pass through the intersection without coming to a stop and driver maybe get to destination without idling. Using a stochastic simulation technique, the velocity planning algorithm has been tested on Veins platform, the results show that the energy/emission savings for vehicles with velocity planning system are found to be $26 \%$ 27\% and the travel time on an average is $1.8 \%$ shorter for the vehicles with velocity planning system as compared to the vehicles without velocity planning system.
\end{abstract}

\section{Introduction}

Along with the rapid development of economy, the number of motor vehicles has a great growth in china, meanwhile the traffic safety problems and environmental pollutions are becoming more and more serious. It's very difficult to solve the increasingly serious traffic safety problems relying on traditional traffic management methods, more and more attention has been paid to improve traffic conditions. Along with the emergency and development of intelligent transport system, all kinds of resources can be used more efficiently and the intellectualized transportation system can be controlled in order [1-3]. Internet of Vehicles is one of the important technologies of intelligent transportation system, it can realize the active information interaction between the vehicle and everything (V2X) around it with wireless communication technologies and image sensor, RFID device, GPS module, the electronic control unit loaded by the body. V2X includes but not limited to: vehicle to vehicle communication (V2V), vehicle to infrastructure communication (V2I), vehicle to pedestrian communication (V2P) and vehicle to Internet communication (V2N). Intelligent vehicle infrastructure cooperative technology is an important part of intelligent transportation system, compared with the passive environment sensing system such as CCD/CMOS image sensor and millimeter wave radar, the active environment perception system can realize the accurate perception of all-weather condition. Drivers with it can avoid traffic accidents, traffic congestion problems caused by the lack of understanding of the driving condition, the inaccurate judgment of the distance of the car and roads, so that fuel and time resources are better used, meanwhile the travel efficiency 
and comfort are improved [4-8] .

V2X communications is based on one of two main technologies: dedicated short-range communication (DSRC) and 4G-LTE. However, in the near future, it is not expected that a single technology can support such a variety of expected V2X applications for a large number of vehicles[9, 10]. Either DSRC or 4G-LTE can't be covered in all directions, especially in mountain area, where the blind area is larger, and intelligent vehicle infrastructure cooperative is more needed. ZigBee plays an important role, mainly in the interconnection of wireless sensor with vehicles and infrastructure. In view of the characteristics low power consumption, short time delay, networking et al, so we focused on the study of intelligent vehicle infrastructure cooperative system based on ZigBee. There are two types of devices in ZigBee wireless network: full-function devices (FFDs) and reduced-function devices (RFDs). The FFD is capable of performing all the duties and can accept any role in the network. The RFD, on the other hand, has limited capabilities. For example, the FFD can communicate with any other devices in a network, but the RFD can talk only with a FFD device. There are three network topology structures that with ZigBee protocol: star, cluster tree and mesh[11]. Many researchers have studied the application of ZigBee in the field of intelligent transportation [12-14]. This paper mainly studies the velocity planning system based on intelligent vehicle infrastructure cooperative technology with ZigBee wireless protocol. With the assistance of this system, the driver doesn't have to worry about whether he/she can make it to the intersection before the traffic light turns red or not. and whether allowed to parking or turn around etc. As a result, unnecessary sharp accelerations or decelerations, illegal parking, illegal turning can be avoided.

\section{System Configuration}

Velocity planning system based on intelligent vehicle infrastructure cooperative technology consists of two units. One is road side unit (RSU), the other is on board unit (OBU). RSU as an infrastructure is installed on the intersection traffic signal lights pole. OBU is installed on vehicle. Either RSU or OBU contains data acquisition module with GPS or Ethernet, data processing module with ARM9 board and data transmission module with ZigBee board.

Data stream between road side unit and on board unit is shown in Fig.1. Vehicles get speed and position by GPS module and transmit to road side unit with ZigBee module. When road side unit gets vehicle's speed, position and the driving direction, with traffic signal light phase and timing got from traffic control platform to give speed advice to the driver such that by the time the vehicle reaches a particular signal, it will be green. Simultaneously, on board unit shows the advice speed and road information such as no parking, no U-turn on LCD screen.

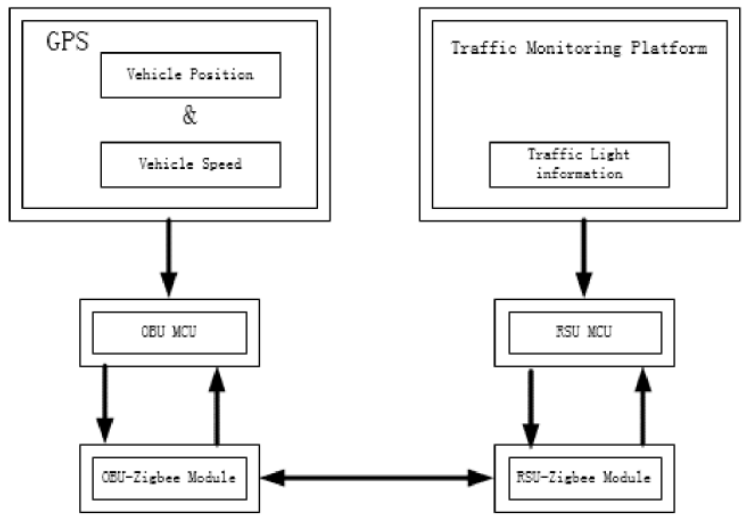

Figure 1. Data Stream

\section{Velocity Planning Algorithm}

The main purpose of velocity planning algorithm is giving dynamic speed advice to the driver so that the probability of having a green light is maximized when approaching signalized intersections. The algorithm is aimed at ensuring that the vehicle never exceeds the speed limit, and that it will 
pass through intersections without coming to a stop. For simplicity, we attribute the yellow phase of traffic signal light to the red phase, and if the traffic signal light phase is red, the vehicle will be able to pass through the intersection at the next green phase.

\subsection{System States}

The algorithm strategy contains: vehicle, intersection and traffic signal light. Distance to the next intersection when OBU gets first acknowledgement from RSU is $s_{p}$, the time $t$ is 0 , and the vehicle velocity is $v_{0}$, the current traffic light phase can be either red or green, as shown in Fig. 2 . If current traffic signal light phase is red then next traffic signal light phase is green followed by red and so on. And if current traffic signal light phase is green then next traffic signal light phase is red followed by green and so on. $t_{r 0}, t_{g 0}, t_{r 1}, t_{g 1}$ is traffic signal light timing sequence, $t_{r 0}, t_{g 0}$ is the time that traffic light turn to red or green first time, and $t_{r 1}, t_{g 1}$ is the time that traffic light turn to red or green once again. $t_{r 0}<t_{g 0}<t_{r 1}$ and $t_{g 0}<t_{r 0}<t_{g 1}$.

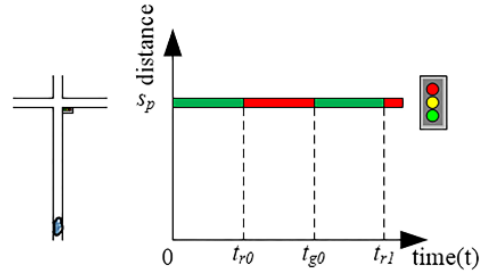

a). Traffic light phase $=$ green

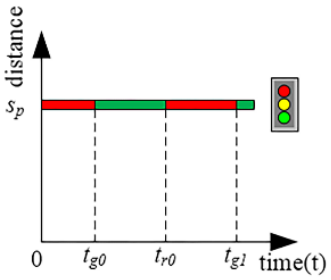

b).Traffic light phase=red

Figure 2. States of traffic light:(a)traffic light phase is green,(b) traffic light phase is red

\subsection{Algorithm Strategy}

The goal of the velocity planning algorithm is that a driver should always get a green signal when she/he reaches a signalized intersection. According to the velocity variation, the vehicle trajectories pass through the intersection can be divided into three types: 1) the vehicle can keep the uniform cruise mode to pass through the intersection, 2) the acceleration mode which the vehicle speeds up again then at a uniform speed pass through the intersection before traffic light phase changes from green to red, 3) the deceleration mode which the vehicle is too late to pass through the intersection, it slows down and then travels at uniform speed so as to pass through the intersection at the next green phase. Fig. 3 shows the vehicle trajectories. "with" means the vehicle is guided by the velocity planning system, and the "without" means that vehicle is not guided by the velocity planning system. Fig. 4 shows the velocity changes with the velocity planning system, while $v_{\max }$ is the limit speed of the road section allowing the vehicle to pass. Which kind of vehicle trajectories the vehicle will choose depends on three factors: the traffic signal light phase and timing in the next intersection, the distance from the vehicle to the intersection, the velocity.
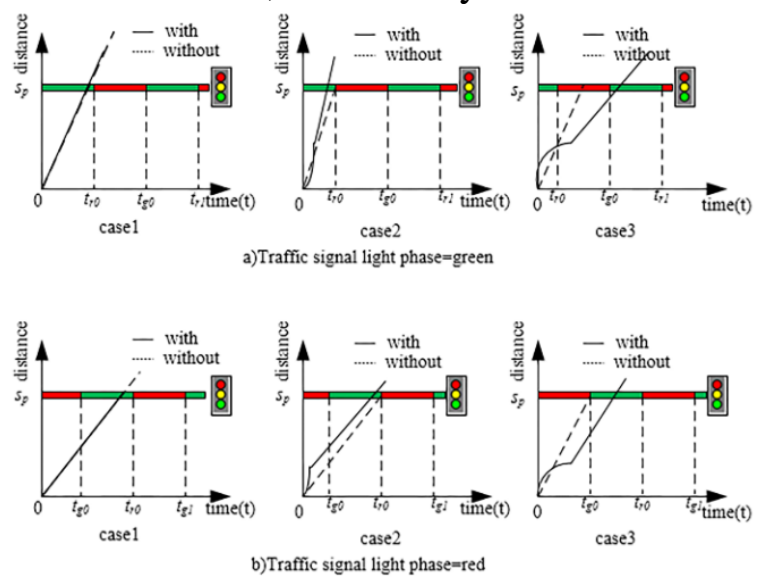

Figure 3. Time-space diagram representing different vehicle trajectories approaching a signalized intersection:(a)traffic light phase is green,(b)traffic light phase is red 


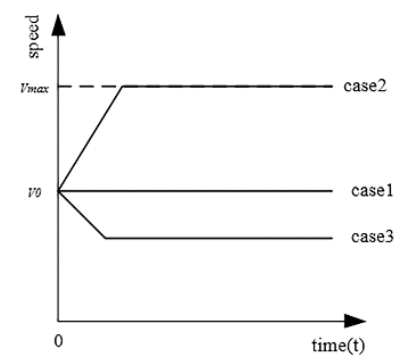

Figure 4. Velocity diagram with different vehicle trajectories.

$t_{p}$ is the time taken to reach the signalized intersection from firstly receive RSU acknowledgement, in order to pass through the intersection at green phase, $t_{p}$ is:

$$
t_{p} \in\left[t_{l}, t_{h}\right] \in\left\{\begin{array}{l}
{\left[\begin{array}{l}
\left.0, t_{r} 0\right) \text { or }\left(t_{g 0}, t_{r}\right), \\
\left(t_{g 0}, t_{r}\right),
\end{array} \quad \text { if traffic signal light phase }=\right.\text { green }} \\
\text { if trignal light phase }=\text { red }
\end{array}\right.
$$

$t_{l}$ and $t_{h}$ are the lower and upper thresholds of $t_{p}$.If the current traffic signal light phase is green , there are two possibilities for $t_{p}$. One is the vehicle pass through the intersection when the light is green, and the $t_{p}$ is $t_{p} \in\left[t_{l}, t_{h}\right]=\left[0, t_{r 0}\right)$; the other is that the velocity of the vehicle is accelerated to the road limit speed $v_{\text {max }}$, but the vehicle can still not pass through the intersection, so it needs to pass through the intersection in the next cycle. At this point, $t_{p}$ is $t_{p} \in\left[t_{l}, t_{h}\right]=\left(t_{g 0}, t_{r 1}\right)$. If the current traffic signal phase is red, then the $t_{p}$ is $t_{p} \in\left[t_{l}, t_{h}\right]=\left(t_{g 0}, t_{r 0}\right)$.

Therefore, the possible velocities of the vehicle fall into a range given by $v_{l o}=s_{p} / t_{h}$ and $v_{h o}=s_{p} / t_{l}$. So the target speed for vehicle to pass through the intersection $v_{p}$ is :

$$
v_{p}=\frac{S_{p}}{t_{p}}=\left[v_{l 0}, v_{h 0}\right] \cap\left[0, v_{\max }\right]=\left[v_{l}, v_{h}\right]
$$

$\mathrm{v}_{l}$ and $v_{h}$ are the lower and upper thresholds of $v_{p}$. In order to stay within the targeted range of velocity, or to achieve a velocity so that the vehicle can reach the intersection at a specific time, the vehicle will need the ability to accelerate or decelerate at specific time, as indicated in Fig.5.

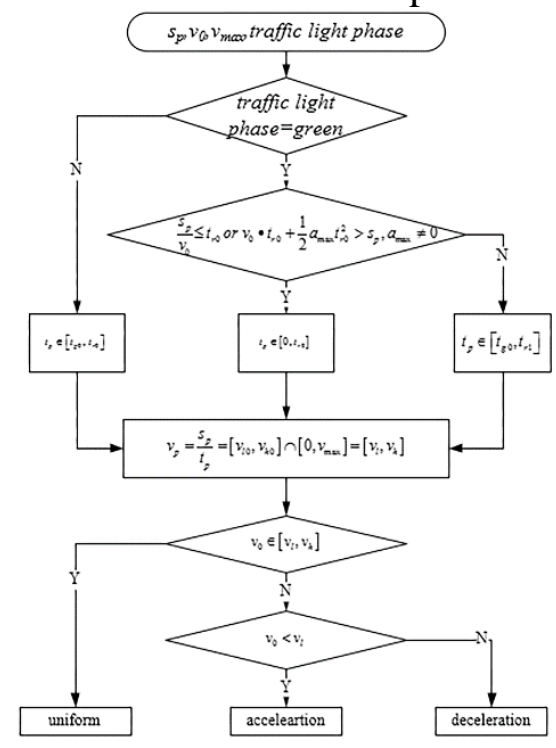

Figure 5. Block Diagram of the velocity algorithm strategy.

\section{Simulation experiment based on velocity planning algorithm}

In this section, we apply the velocity planning system presented in previous section to generate 
vehicle velocity profiles of a vehicle traversing a hypothetical 10-signalized intersection corridor, as shown in Fig. 6 and use stochastic simulation to capture the variability of vehicle-related parameters.

\subsection{Choose Simulate Platform}

Veins is an open source framework for running vehicular network simulations. It is based on two well-established simulators: OMNet++, an event-based network simulator, and SUMO, a road traffic simulator. Both simulators are connected via a TCP socket. The protocol for this communication has been standardized as the Traffic Control Interface (TraCI). This allows directionally-coupled simulation of road traffic and network traffic. It extends these to offer a comprehensive suite of models for inter-vehicular communication simulation[15].

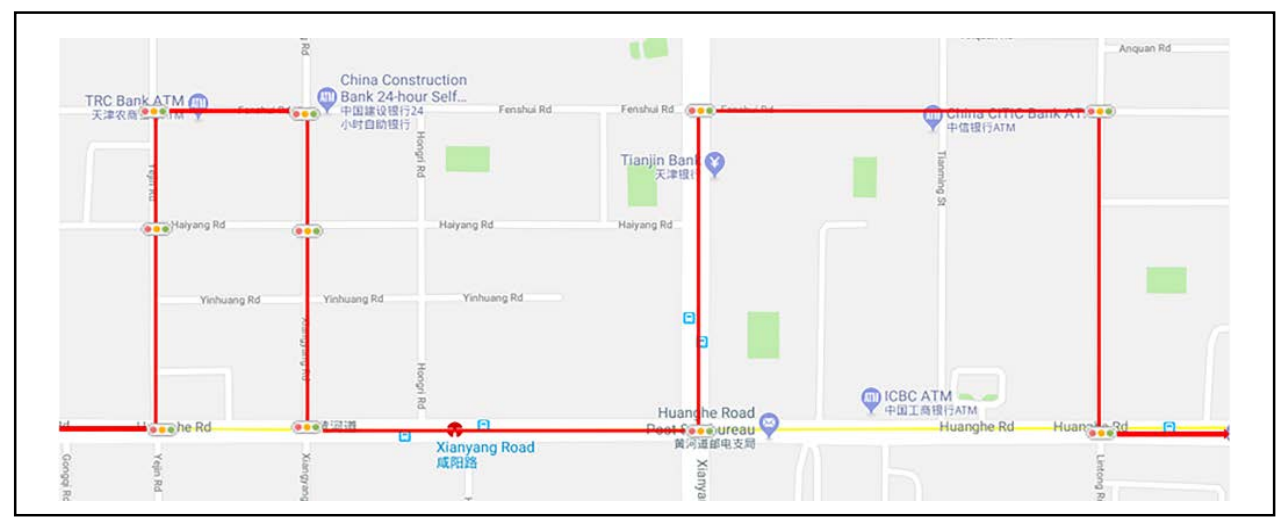

Figure 6. Route for simulationSimulation Setup

In this paper, RSU and OBU exchange information with ZigBee wireless technology. In order to prevent collisions between different device nodes in the network, carrier sense multiple access/collision avoidance (CSMA/CA) mechanism was used in the simulation. Table 1 shows the parameters of ZigBee in the simulation and Table 2 shows the parameters for simulation. The intersection layout in the simulation model is shown in Fig. 7. The simulation was performed for a typical mid-sized sedan car.

Table 1 Zigbee protocol parameters.

\begin{tabular}{|c|c|}
\hline Parameter & Value \\
\hline PHY model & IEEE802.15.4 \\
\hline Carrier Frequency & $2.49 \mathrm{GHz}$ \\
\hline Max transmission power & $1.1 \mathrm{~mW}$ \\
\hline Signal attenuation threshold & $-100 \mathrm{dBm}$ \\
\hline Path loss coefficient alpha & 2.5 \\
\hline Sensitivity & $-100 \mathrm{dBm}$ \\
\hline
\end{tabular}

Table 2 The parameters for simulation

\begin{tabular}{|c|c|}
\hline Parameter & Value \\
\hline length of road & $20 \mathrm{~km}$ \\
\hline limit speed & $60 \mathrm{~km} / \mathrm{h}$ \\
\hline R-duration & $30 \mathrm{~s}$ \\
\hline G-duration & $27 \mathrm{~s}$ \\
\hline Y-duration & $3 \mathrm{~s}$ \\
\hline vehicle density & $10 / \mathrm{km}$ \\
\hline Vehicle acceleration & $2 \mathrm{~m} / \mathrm{s}^{2}$ \\
\hline Time step & $10 \mathrm{~ms}$ \\
\hline
\end{tabular}




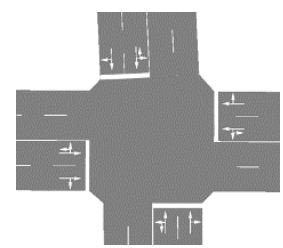

Figure 7. 'i ne intersecuon rayout in tne sımuiatıon.

\subsection{Simulation Result}

For comparison purposes, the speed, the emissions and fuel consumptions for those vehicles that without velocity planning system are also calculated for the same vehicle categories. Table 3 shows the standard deviation of speed is reduced by $68 \%$ compared with those without velocity planning system. It shows that there is no sudden acceleration and deceleration in the driving process of the vehicle. Meanwhile the minimum speed of vehicle isn't zero so there is no stopping. Fig. 8 and Fig. 9 respectively shows the distance-time diagram of the vehicle as well as the traffic signal light phase and timing at each intersection for that simulation run with and without velocity planning system. As we can see from them that the travel time on an average is $1.8 \%$ shorter for the vehicles with velocity planning as compared to the vehicles without velocity planning.

Table 3 Velocity statistics.

\begin{tabular}{|c|c|c|c|c|}
\hline $\begin{array}{c}\text { Vehicle } \\
\text { speed }\end{array}$ & $\begin{array}{c}\text { Min } \\
(\mathrm{km} / \mathrm{h})\end{array}$ & $\begin{array}{c}\text { Max } \\
(\mathrm{km} / \mathrm{h})\end{array}$ & $\begin{array}{c}\text { Mean } \\
(\mathrm{km} / \mathrm{h})\end{array}$ & $\begin{array}{c}\text { StdDev } \\
(\mathrm{km} / \mathrm{h})\end{array}$ \\
\hline Without & 0 & 59.528 & 35.538 & 11.449 \\
\hline With & 9.921 & 43.745 & 35.467 & 3.651 \\
\hline
\end{tabular}

Table 4 shows the energy and emissions comparison results between the vehicles with and without IVICS. According to Table 4, the vehicles with velocity planning system consume about $27 \%$ less fuel and produce about 26.4\% less CO2 emission.

Table 4 Results of Pollutant Emissions and Fuel Consumption.

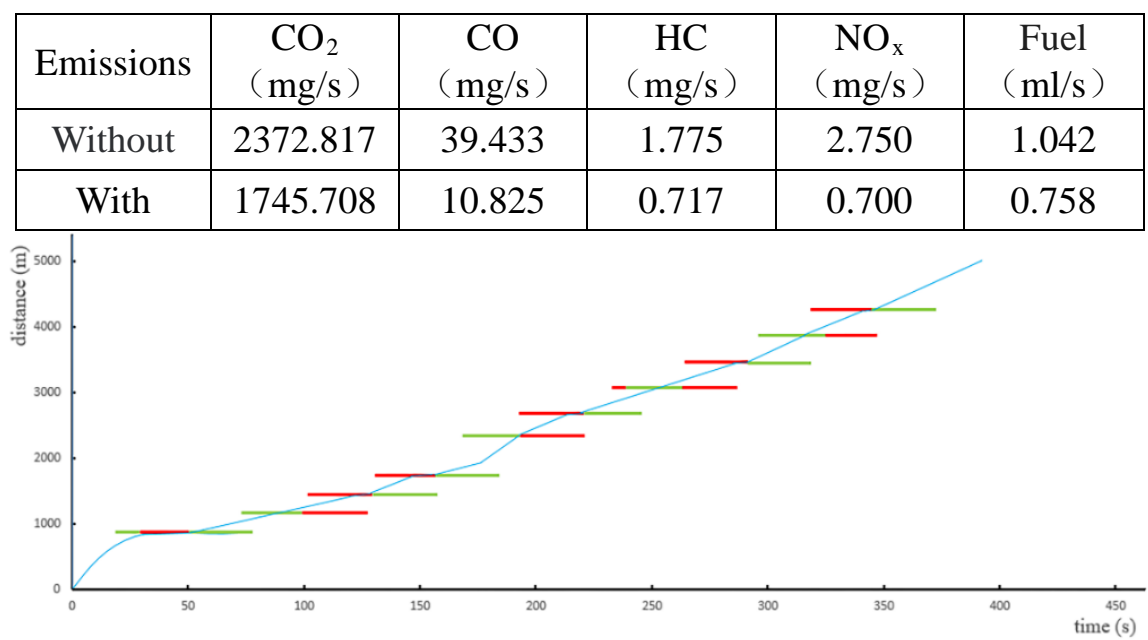

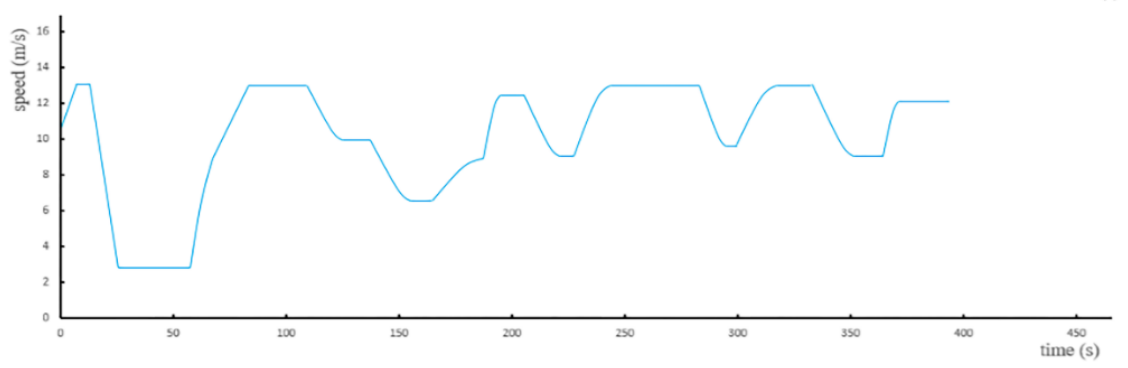

Figure 8. Sample trajectory of vehicle with velocity planning system. 

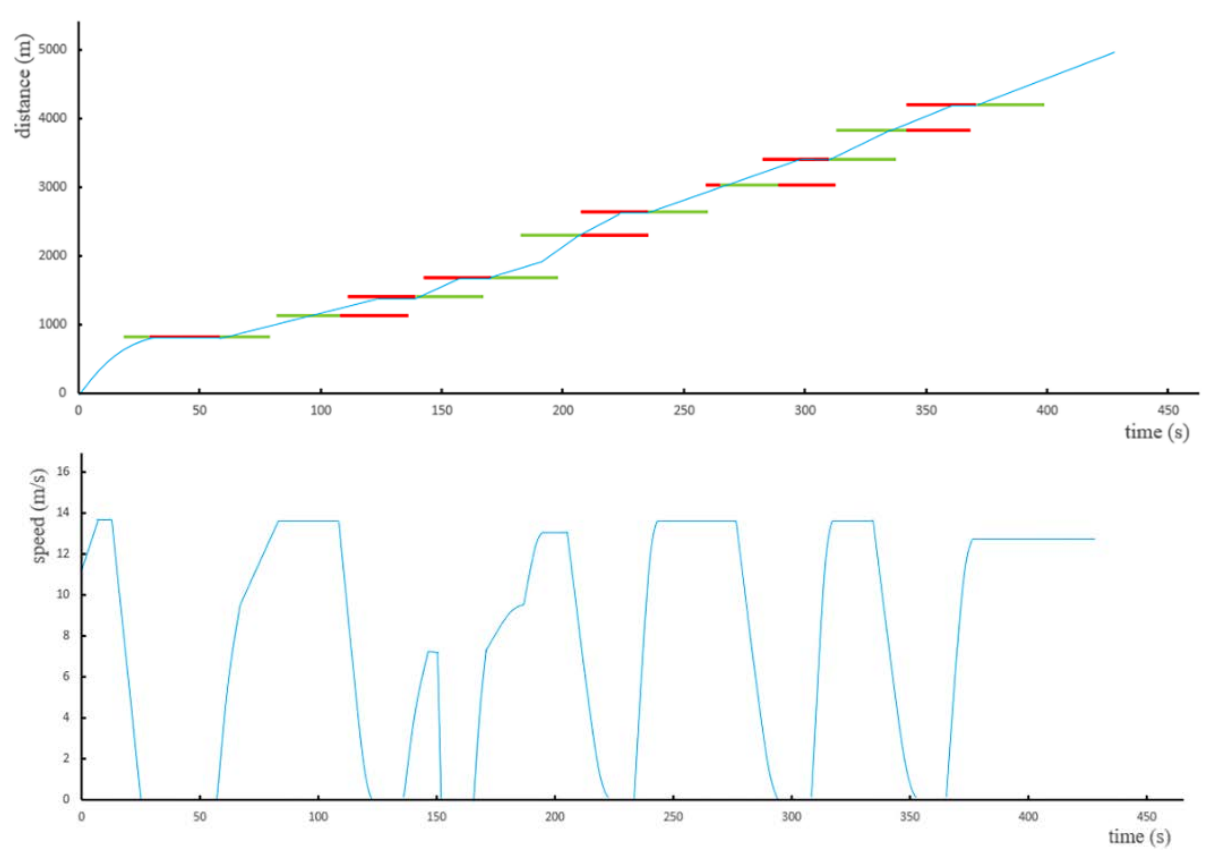

Figure 9. Sample trajectory of vehicle without velocity planning system.

\section{Conclusions}

By taking advantage of the ZigBee technology, it is possible to obtain the traffic signal light phase and timing information in situ. Based on the real-time traffic signal light information, we have initially developed an algorithm that can provide dynamic speed advice to the driver such that the driver can adjust his/her speed so that the probability of passing through a signalized intersection without stopping is maximized. In addition, the speed transitions can be made smoothly to minimize emissions from sharp accelerations or decelerations. Based on the algorithms presented in this paper, the energy/emission savings for vehicles with velocity planning system are found to be $26-27 \%$ and the travel times is $1.8 \%$ shorter than those without velocity planning system.

\section{References}

[1] Gupta P, Purohit G N, A D, "Approaches for intelligent traffic system: A survey", International Journal on Computer Science \& Engineering, vol.4, no.9, pp.1570-1578,2012.

[2] Hamida E, Noura H, Znaidi W, "Security of cooperative intelligent transport systems: Standards, threats analysis and cryptographic countermeasures", Electronics, vol.4, no.3, pp.380-423,2015.

[3] Alam KM, Saini M, Saddik AE, "Toward social internet of vehicles: Concept, architecture, and applications", IEEE Access,vol.3,pp.343-357,2015.

[4] Wedel, Jan W, Radusch I, "V2x-based traffic congestion recognition and avoidance", International Symposium on Pervasive Systems, Algorithms, and Networks, vol.13, pp.637641,2010 .

[5] Lefevre S, Petit J, Bajcsy R, Laugier C, Kargl F, "Impact of v2x privacy strategies on intersection collision avoidance systems", Vehicular Networking Conference,pp.71-78,2014.

[6] Li J, Dridi M, El-Moudni, "A cooperative traffic control of vehicle-intersection (ctcvi) for the reduction of traffic delays and fuel consumption", Sensors (Basel), vol.16, no.2175, pp.1-20,2016.

[7] Krzysztof Małecki SI, Kinga Kijewska, "Influence of intelligent transportation systems on reduction of the environmental negative impact of urban freight transport based on szczecin example", Procedia - Social and Behavioral Sciences vol.151,pp.215-229,2014.

[8] VivekN SVS, RajuK,etal, "Safety alert \& advisory information system using vehicular 
communication (saver)". In: IEEE, editor. IEEE International Conference on Recent Advances and Innovations in Engineering; Jaipur, India. 2014.

[9] Abboud K, Omar H A, W Z, "Interworking of dsrc and cellular network technologies for v2x communications: A survey", IEEE Transactions on Vehicular Technology,vol.65,no.12,pp.94579470,2016 .

[10] Vukadinovic V, Bakowski K, Marsch P, Garcia ID, Xu H, Sybis M, et al., "3gpp c-v2x and ieee 802.11p for vehicle-to-vehicle communications in highway platooning scenarios", Ad Hoc Networks,vol.74,pp.17-29,2018.

[11] Farahani S. Zigbee wireless networks and transceiver. United Kingdom: Newnes 2008.

[12] Biaou US, Laid \& Bocquet, Michael \& Menhaj, Atika., "Modeling of zigbee (ieee 802.15.4) channel in rail environment for intelligent transport". International Conference on Advanced Logistics and Transport Valenciennes, France293-298. 2015.

[13] Selvarajah K, Tully A, Blythe PT, "Zigbee for intelligent transport system applications". Road Transport Information and Control - RTIC 2008 and ITS United Kingdom: United Kingdom Members' Conference, IET, p. 1-7. 2008.

[14] Zhou YU, Weizeng GAO, ZUO X, "Design of novel intelligent transportation system based on wireless sensor network and zigbee technology", Sensors \& Transducers,vol.156,no.9,pp.95$102,2013$.

[15] Sommer C, German R, Dressler F, "Bidirectionally coupled network and road traffic simulation for improved ivc analysis", IEEE Transactions on Mobile Computing,vol.10,no.1,pp.315,2010 . 